\title{
Ventral hernia and diastasis recti. Is there a consensus?
}

\author{
G. Campanelli ${ }^{1}$
}

Published online: 29 July 2021

(c) The Author(s), under exclusive licence to Springer-Verlag France SAS, part of Springer Nature 2021

Diastasis recti remains poorly understood in terms of the physio-pathogenesis and anatomical aspects of the disorder, as well as the therapeutic indications and possible surgical approaches.

Physiotherapists, plastic surgeons, general surgeons, and hernia repair specialists, who periodically propose different solutions, are the key players in this field.

Ventral hernia and diastasis recti, given the many aspects needing to be considered, are not easy problems to address, and they have no single solution.

First of all, there is the ethical aspect, by which we mean the need to establish, with absolute transparency, the correct therapeutic indication.

As a result of the growing culture of "DIY healthcare" on the internet, there are now "armies" of would-be abdominal wall experts-self-styled "surgical team directors" with no real acquired expertise, no history in the field, and no official academic or professional recognition. These are individuals who, being in a position to buy advertising space and visibility on social networks, are able to present themselves as "healers" of diastasis and ventral hernias. With their persuasive approach, they can manage to win over the most ingenuous and least informed patients, only to then run into complication upon complication.

In surgery it is fundamental, for the good of the patient, to practise sound ethics, which means being honest about the correct indication.

Since diastasis recti is a controversial topic, the ethical aspect can, in this case, be open to different and sometimes incorrect interpretations.

The second critical aspect is the therapeutic approach. At present there is still no standardised approach, meaning that it is left to individual professionals to decide whether to opt for physio-kinesitherapy, surgery or a combined treatment.

G. Campanelli

hernia.editorialoffice@gmail.com

1 University of Insubria, Gruppo Ospedaliero San Donato, Milan, Italy
And when the surgical approach is chosen, this prompts a further series of questions. What surgery would be best? Minimally invasive open, laparoscopic, endoscopic, robotic? With or without a prosthesis? What type of prosthesis? With or without concomitant aesthetic interventions (abdominoplasty or mini-abdominoplasty)? In short, the open questions are numerous.

In our view, it is crucial to go back to trying to evaluate the pathophysiology, the risk factors, and the anatomical preconditions - all elements that, taken together, can guide us towards a reasoned and reasonable choice, and allow us to propose, in good conscience, the correct option for the individual case.

In this issue of HERNIA, we have tried to contribute to the debate on this increasingly "trendy" topic. With the authoritative support of David Ross, Maurice Nahabedian, Jeffrey Janis and Gregory Dumanian as guest editors, we have put together an update that we hope will be of help in the future development and implementation of guidelines.

LATEST NEWS: Hernia 2020 impact factor has reached 4.739 .

Impact Factor

\begin{tabular}{|l|l|l|}
$\begin{array}{l}4.739 \\
2020\end{array}$ & $\mathbf{3 . 6 9 4}$ \\
5 year & \\
\hline JCR @ Category & Rank in Category & Quartile in Category \\
\hline SURGERY & $\mathbf{2 7}$ of 212 & $\mathbf{Q 1}$ \\
\hline
\end{tabular}

Data from the 2020 edition of Journal Citation Reports

Publisher's Note Springer Nature remains neutral with regard to jurisdictional claims in published maps and institutional affiliations. 